\title{
Notch4 inhibition reduces migration and invasion and enhances sensitivity to docetaxel by inhibiting Akt/fascin in pancreatic cancer cells
}

\author{
CUI-JUAN QIAN ${ }^{1,2^{*}}$, YI-YI CHEN ${ }^{1 *}$, XIN ZHANG $^{2}$, FU-QIANG LIU ${ }^{1}$, TING-TING YUE ${ }^{1}$, BEI YE ${ }^{2}$ and JUN YAO ${ }^{1}$ \\ ${ }^{1}$ Institute of Tumor, School of Medicine, Taizhou University; ${ }^{2}$ Department of Gastroenterology, \\ Taizhou University Affiliated Taizhou Municipal Hospital, Taizhou, Zhejiang 318000, P.R. China
}

Received June 18, 2015; Accepted August 19, 2016

DOI: $10.3892 / \mathrm{ol} .2016 .5097$

\begin{abstract}
Overexpression of Notch4 is associated with a variety of tumor types. Only sparse information exists on Notch4 expression in pancreatic cancer (PC). The present study demonstrated that Notch4 expression was significantly upregulated in PC cell lines compared with a non-transformed pancreatic epithelial cell line, HPDE6c-7. To investigate the possible role of Notch4 in PC cells, an RNA interference approach was used to silence Notch4 expression. The results revealed that small interfering RNA (siRNA) targeting Notch4 significantly impeded the viability, migration and invasion abilities of PC cells in vitro. Downregulation of Notch4 with siRNA sensitized cells to the action of docetaxel. Furthermore, Notch4 downregulation enhanced the inhibition of Akt activation and the fascin expression induced by docetaxel in PC cells. Together, these data provide insight into the function of Notch4 and suggest that Notch4 may represent a new potential target for gene therapy in $\mathrm{PC}$.
\end{abstract}

\section{Introduction}

In spite of a major decline in incidence and mortality over several decades, pancreatic cancer (PC) is still one of the most common causes of cancer mortality worldwide (1-3). Since PC is a tumor with a high potential of invasion and

Correspondence to: Professor Jun Yao, Institute of Tumor, School of Medicine, Taizhou University, 1139 Shifu Road, Jiaojiang, Taizhou, Zhejiang 318000, P.R. China

E-mail: yaojuntzu@yeah.net

${ }^{*}$ Contributed equally

Abbreviations: PC, pancreatic cancer; RNAi, RNA interference; siRNA, small interfering RNA; MTT, 3-(4,5-dimethylthiazol-2-yl)2,5-diphenyltetrazolium bromide

Key words: Notch4, pancreatic cancer, siRNA, cell viability, metastasis metastasis, the majority of patients with PC are in advanced stage when they are diagnosed (4). Although numerous studies indicate that tumor invasion and metastasis are attributed to the increase in cell motility and loss of cell-cell adhesion (5), the molecular mechanisms of PC invasion and metastasis are not completely understood.

Notch4, which belongs to a family of transmembrane receptor proteins (Notch1 to Notch4), is upregulated in numerous malignant tumors and plays critical roles in various cell fate decisions, including proliferation, apoptosis and differentiation (6-9). Previous studies demonstrated that Notch4 is overexpressed in human hepatocellular carcinoma and is associated with poor overall survival (8), and that inactivation of Notch4 by small interfering RNA (siRNA) sensitizes breast tumor cells to TNF-related apoptosis-inducing ligand-induced apoptosis (7). Furthermore, our previous study demonstrated that Notch 4 contributes to gastric cancer cell growth associated with Wnt $1 / \beta$-catenin activation (6). These data suggest that Notch4 is important in tumorigenesis, and that Notch4 may be a potential target for cancer prevention and therapy.

The serine/threonine kinase Akt, also known as protein kinase $\mathrm{B}$, is a central node in cell signaling downstream of growth factors, cytokines and other cellular stimuli (10). Aberrant Akt activation is one of the most common molecular alterations in cancer and contributes to tumorigenesis (10), as well as to promote resistance to chemotherapy (11-13). In addition, Akt is a potential target for enhancing DNA damage or apoptosis in response to chemotherapeutic agents in cancer therapy (11-13). Since there may be cross-talk between Notch4 and Akt (14), we expect that Notch4 may regulate chemosensitivity via Akt in $\mathrm{PC}$ cells.

However, to date, there are no additional studies with respect to the role of Notch4 in pancreatic carcinogenesis. In order to investigate the role of Notch4 in pancreatic carcinogenesis, siRNA was used in the present study to specifically inhibit Notch4 expression in several PC cell lines, and cell viability, migration and invasion of $\mathrm{PC}$ cells were detected in vitro. Furthermore, the potential induction of chemosensitivity in PC cells by a chemotherapeutic agent under treatment with Notch4 siRNA was also investigated. 


\section{Materials and methods}

Cell culture. The human PC cell lines MiaPaCa-2, BxPC3, PANC-1 and Capan-1, as well as HPDE6c7, an immortalized but not transformed pancreatic epithelial cell line, were cultured in 90\% RPMI 1640 (Gibco; Thermo Fisher Scientific, Inc., Waltham, MA, USA) supplemented with antibiotics (1X penicillin/streptomycin $100 \mathrm{U} / \mathrm{ml}$ ) and $10 \%$ fetal bovine serum (FBS) (Gibco; Thermo Fisher Scientific, Inc.). Cells were incubated in a humidified atmosphere containing $5 \% \mathrm{CO}_{2}$ at $37^{\circ} \mathrm{C}$.

Western blot analysis. Western blot analysis was performed to evaluate the protein levels of Notch4, phosphorylated (p)-Akt, Akt, glycogen synthase kinase 3 (GSK3), p-GSK3 and fascin. Proteins were harvested from cells using $2 \mathrm{X}$ electrophoresis sample buffer [125 mmol/1 Tris- $\mathrm{HCl}$ (pH 6.8), 2\% sodium dodecyl sulfate (SDS), $5 \%$ glycerol and $1 \% \beta$-mercaptoethanol]. Equal amounts of denatured proteins $(35 \mu \mathrm{g})$ were resolved by $10 \%$ SDS-polyacrylamide gel electrophoresis and then blotted onto polyvinylidene fluoride membranes (Pall Life Sciences, Port Washington, NY, USA). After blocking for $2 \mathrm{~h}$ with phosphate-buffered saline containing $0.1 \%$ Tween 20 (PBST) and $5 \%$ powdered skim milk, the blots were incubated with primary Notch4 (cat.no.ab134831; 1:1,000),p-Akt (cat.no. ab8933; 1:500), Akt (cat. no. ab8805; 1:1,000), GSK3 (cat. no. ab131344; 1:500), p-GSK3 (cat. no. ab28808; 1:500) and fascin (cat. no. ab97753; 1:1,000) antibodies (Abcam, Cambridge, UK) overnight in 5\% powdered skim milk buffer, washed thrice with PBST and incubated with horseradish peroxidase-conjugated anti-rabbit (cat. no. 7074) and anti-mouse (cat. no. 7076) IgG secondary antibodies (1:2,000; Cell Signaling Technology, Beverly, MA, USA). An anti-glyceraldehyde 3-phosphate dehydrogenase antibody (cat. no. sc-25778; 1:1,000; Santa Cruz Biotechnology, Inc., Dallas, TX, USA) was used as a control. All bands were detected using ECL Western Blotting (GE Healthcare Life Sciences, Chalfont, UK).

siRNA transfection. The siRNAs targeting specific sequences of Notch4 and a negative control scrambled siRNA (not homologous to any gene) were synthesized by Shanghai GenePharma Co., Ltd. (Shanghai, China) (6). The PC cells were seeded into 6 -well plates $\left(4-5 \times 10^{4}\right.$ cells/well) and cultured in $2 \mathrm{ml}$ of basic culture medium containing 10\% FBS until the cells were $70 \%$ confluent. Cells were transfected with control (non-specific siRNA) or specific siRNA (100-200 nM) for $48 \mathrm{~h}$.

\section{3-(4,5-Dimethylthiazol-2-yl) 2,5-diphenyltetrazolium} bromide (MTT) assay. Untransfected PC cells (blank control) and PC cells $\left(1 \times 10^{6}\right)$ transfected with Notch4 siRNA or control siRNA in $200 \mu 1$ RPMI 1640 were seeded in duplicate into each well of a 96-well culture plate, and $100 \mu \mathrm{l}$ MTT $(5 \mathrm{mg} / \mathrm{ml}$; Sigma-Aldrich; Merck Millipore, Darmstadt, Germany) was added at 24, 48, 72, 96, 120, 144 and $168 \mathrm{~h}$. After 4-h incubation at $37^{\circ} \mathrm{C}$ in $5 \% \mathrm{CO}_{2}, 100 \mu \mathrm{l}$ dimethyl sulfoxide was added to solubilize the formazan product for $30 \mathrm{~min}$ at room temperature. The absorbance at $570 \mathrm{~nm}$ was determined using a microplate reader (Bio-Rad Laboratories, Inc., Hercules, CA, USA).

Cell migration and invasion assays. Cell migration was assessed using transwell permeable supports (Corning Inc.,
Corning, NY, USA). Briefly, cells were allowed to grow to confluence. In total, $5 \times 10^{4}$ cells/well were resuspended in $100 \mu \mathrm{l}$ serum-free medium and plated onto uncoated $8-\mu \mathrm{m}$ transwell filter inserts in 24-well plates in triplicate. The lower chambers contained $600 \mu \mathrm{l}$ of medium containing $15 \%$ FBS as a chemoattractant. Non-migratory cells in the upper chamber were removed with a cotton swab following incubation for $16 \mathrm{~h}$. Cells on the bottom side were fixed in $100 \%$ methanol and stained with $0.5 \mu \mathrm{g} / \mathrm{ml}$ 4',6-diamidino-2-phenylindole for $5 \mathrm{~min}$. Cells were counted using a fluorescence microscope (Eclipse 80i; Nikon Corporation, Tokyo, Japan) in five random fields. For evaluation of cell invasion, cells were allowed to invade matrigel-coated transwell filters. At the end of the experiments, the invaded cells on the bottom of the membrane were incubated with $0.1 \%$ crystal violet solution and dissolved in $20 \%$ acetic acid. Finally, $100 \mu$ l dye mixture was transferred to a 96 -well plate for absorbance readings at $560 \mathrm{~nm}$.

Drug sensitivity assay. To assess the chemosensitivity to docetaxel (Sigma-Aldrich; Merck Millipore), untransfected PC cells (blank control) and PC cells $\left(1 \times 10^{4}\right)$ transfected with Notch 4 siRNA or control siRNA and cultured for $24 \mathrm{~h}$ were incubated with different concentrations of docetaxel $(0,100$, 200 and $400 \mathrm{nM}$ ) for additional $48 \mathrm{~h}$. Then, cells were treated with MTT as described earlier. Each group contained five wells.

Statistical analysis. SPSS 18.0 software (SPSS Inc., Chicago, IL, USA) was used for statistical analysis. The data were expressed as the mean \pm standard deviation, and the Student's $t$-test was used to determine the significance of differences between two groups. $\mathrm{P}<0.05$ was considered to indicate a statistically significant difference.

\section{Results}

Expression of Notch4 in PC cell lines. The expression of Notch4 in the PC cell lines MiaPaCa-2, BxPC3, PANC-1 and Capan-1, as well as in HPDE6c7, an immortalized but not transformed pancreatic epithelial cell line, was determined, and it was observed that, although Notch4 is differentially expressed in PC cell lines, Notch4 expression was significantly upregulated in PC cell lines compared with the normal pancreatic epithelial cell line HPDE6c7, suggesting a correlation of Notch4 expression with pancreatic tumorigenesis and malignancy. In addition, Notch4 expression was higher in MiaPaCa-2, PANC-1 and BxPC3 cells than that in HPDE6c7 and Capan-1 cells (Fig. 1).

Silencing of Notch4 in PC cells by RNA interference (RNAi) approach. To directly test whether silencing of Notch4 was responsible for the decreased proliferative and invasive capabilities of PC cells, Notch4 expression was downregulated in MiaPaCa-2 and PANC-1 cells using an RNAi approach. The protein levels of Notch4 were significantly reduced in Notch4 siRNA-transfected cells after transfection for $48 \mathrm{~h}$ compared with those in the control siRNA or blank control groups (Fig. 2). These results confirmed that Notch4 expression was indeed knocked down by Notch4 siRNA in the PC cells. 


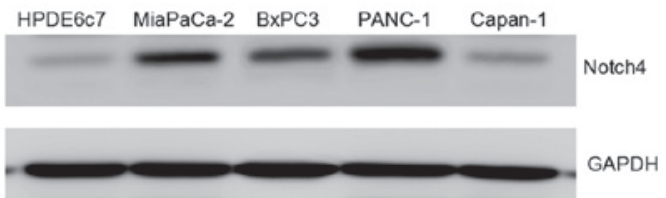

Figure 1. Analysis of Notch4 expression in human PC cell lines. The Notch4 expression levels in cellular total protein of PC cell lines and in HPDE6c7 cells were determined by western blotting. Whole-cell extracts of HPDE6c7, MiaPaCa-2, BxPC3, PANC-1 and Capan-1 cells were used for western blot analysis. The level of GAPDH was used as an internal control. The data were obtained from three independent experiments. PC, pancreatic cancer; GAPDH, glyceraldehyde 3-phosphate dehydrogenase.

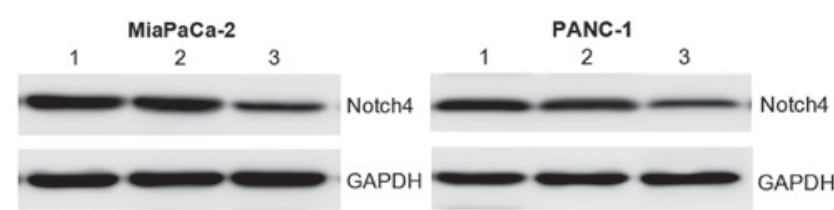

Figure 2. Knockdown of Notch4 in pancreatic cancer cells. Western blot analysis revealed that the expression of Notch4 was highly reduced in Notch4 siRNA-transfected MiaPaCa-2 and PANC-1 cells. GAPDH was used as an internal control to demonstrate equal protein loading. Lane 1, blank control; lane 2, control siRNA; lane 3, Notch4 siRNA. The data were obtained from three independent experiments. GAPDH, glyceraldehyde 3-phosphate dehydrogenase; siRNA, small interfering RNA.

Silencing of Notch4 reduces $P C$ cell viability, migration and invasion in vitro. The cell viability of Notch4-deficient cells was determined by MTT assay. The results indicated that silencing of Notch4 significantly suppressed cell viability as compared with the controls (Fig. 3A). Meanwhile, the cell viability of MiaPaCa-2 and PANC-1 cells transfected with control siRNA was not affected (Fig. 3A). Thus, silencing of Notch4 could inhibit the cell viability of MiaPaCa-2 and PANC-1 cells in vitro.

To examine whether the targeted downregulation of Notch4 in MiaPaCa-2 and PANC-1 cells affected the cell migration and invasion abilities, cell migration and invasion assays were performed. The results of the cell migration (Fig. 3B and C) and invasion (Fig. 3D) assays revealed that the blank control and the control siRNA cells had a similar migration and invasion ability, while cells subjected to Notch4 siRNA transfection migrated and invaded less efficiently compared with the blank control and control siRNA cells. Representative results of cell migration assays are shown in Fig. 3C.

Silencing of Notch4 enhances the sensitivity of PC cells to docetaxel treatment. To examine whether blockade of Notch4 signaling with Notch4 siRNA potentiated the ability of docetaxel to inhibit the cell viability of $\mathrm{PC}$ cells, MiaPaCa-2 and PANC-1 cells transfected with Notch4 siRNA or control siRNA cultured for $24 \mathrm{~h}$ were incubated with different concentrations of docetaxel $(0,100,200$ and $400 \mathrm{nM})$ for additional $48 \mathrm{~h}$. It was observed that treatment with Notch 4 siRNA alone resulted in no or moderate anti-tumor effects (Fig. 4). The cell viability of Notch4 siRNA-transfected cells was markedly reduced with the addition of docetaxel relative to that of the controls, and this reduction was concentration dependent (Fig. 4). In addition, Notch4 siRNA significantly enhanced the inhibition of cell migration (Fig. 3B and C) and invasion (Fig. 3D) induced by docetaxel in MiaPaCa-2 and PANC-1 cells (Fig. 3). Thus, silencing of Notch4 could increase the sensitivity of PC cells to docetaxel treatment.

Downregulation of Notch4 enhances the inhibition of fascin expression and the activation of Akt in PC cells subjected to docetaxel treatment. To elucidate the molecular mechanism by which Notch4 silencing induces cells invasion, western blot analysis was used to examine the expression of fascin, a critical hallmark of the invasive phenotype of cancer cells (15). In MiaPaCa-2 and PANC-1 cells, docetaxel or Notch4 siRNA led to a significant decrease in the expression of fascin, an actin-binding protein that provides mechanical support and cell motility and is involved in PC metastasis (15), which was consistent with the inhibition of cell invasion. Furthermore, the combination of Notch4 siRNA and docetaxel treatment decreased the expression of fascin in MiaPaCa-2 and PANC-1 cells to a much lower level than that in cells treated with Notch4 siRNA or docetaxel alone (Fig. 5).

The Akt family of proteins integrates a wide array of diverse upstream survival and distress signals to decide cell fate (16). GSK3 is a primary target of Akt (17). To investigate the mechanism by which Notch4 silencing regulates cell viability, migration and invasion in MiaPaCa-2 and PANC-1 cells, the protein expression and activation levels of Akt and GSK3 were examined. Compared with cells treated with Notch4 siRNA or docetaxel alone, the results of protein expression of Akt and GSK3 and activation of Akt and GSK3 indicated that p-Akt and p-GSK were significantly downregulated in cells treated with both Notch4 siRNA and docetaxel (Fig. 5). These data suggest that Notch4 siRNA may enhance the docetaxel-induced anti-tumor effects by modulating Akt, GSK3 and fascin in PC cells.

\section{Discussion}

One of the primary aims of tumor research is to identify signaling pathways and genetic factors that contribute to tumorigenesis and to elucidate the mechanism of tumor development (18). In this way, specific proteins can be selected as potential markers for tumor development or as potential targets for tumor therapy. Notch4 signaling has gained attention due to its activation in a number of different tumors (6-8), and Notch4 signaling is involved in the progression of tumors; thus, modulation of this cascade has potential for targeted therapy (6-8). The present study clearly demonstrated that Notch4 expression was higher in human PC cell lines compared with that in a non-transformed pancreatic epithelial cell line. Thus, it is likely that Notch4 is related to the malignant behavior of PC.

Substantial research has been conducted at the molecular level, which is expected to identify clues for markers of PC progression, predictors of outcome and targets for pharmacological therapy (1-3). Notch4 has been shown to influence the cell proliferation of salivary adenoid cystic carcinoma, triple-negative breast cancer and gastric carcinoma (GC) cells $(6,19,20)$. In addition, our previous findings revealed that the activation of Notch4 promoted GC cell growth in vitro and in vivo, while the inactivation of Notch4 by siRNA had the opposite effects (6). In the present study, it was demonstrated 
A

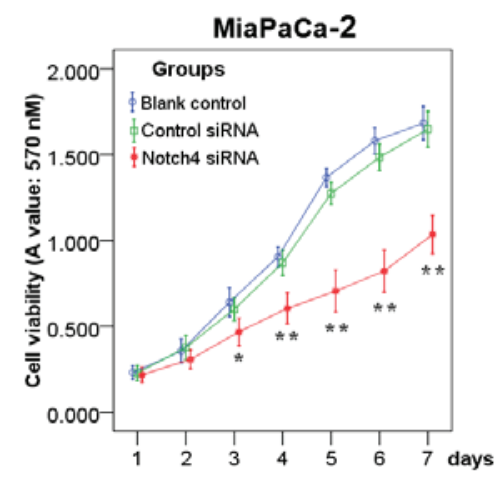

B

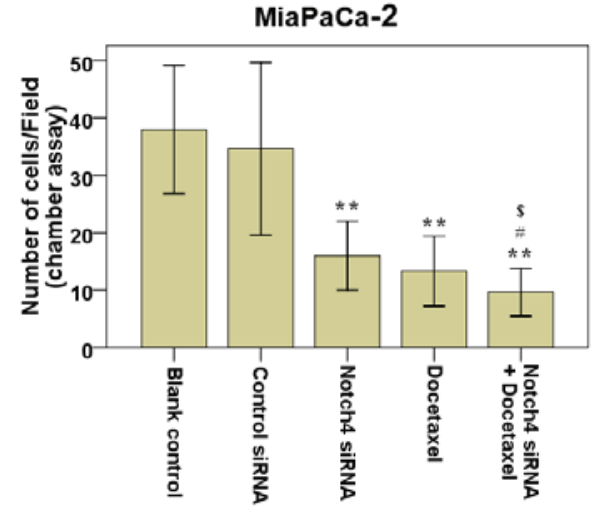

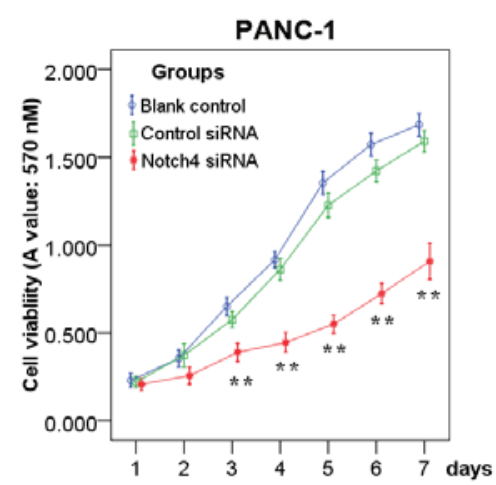

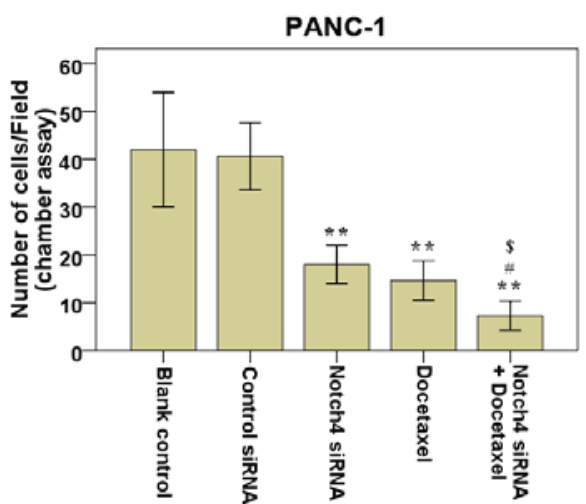

C

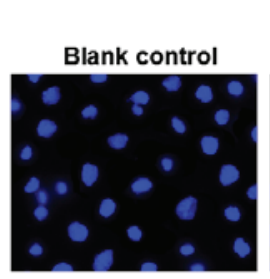

Control SIRNA

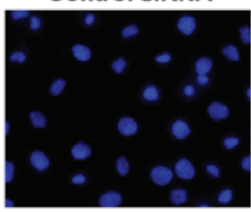

MiaPaCa-2

Notch4 siRNA

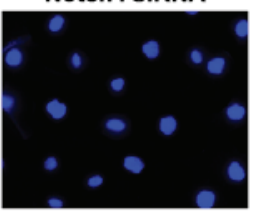

Docetaxel

Notch4 siRNA

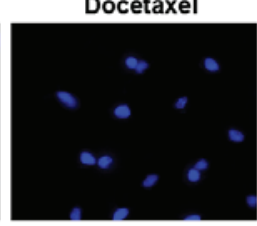

+ Docetaxel

PANC-1

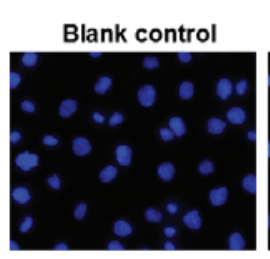

Control siRNA

Notch4 SiRNA
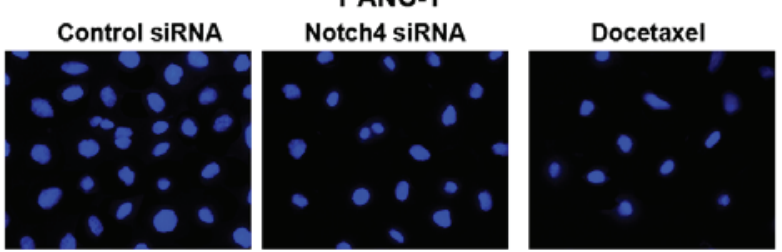

Notch4 siRNA

+ Docetaxel

D
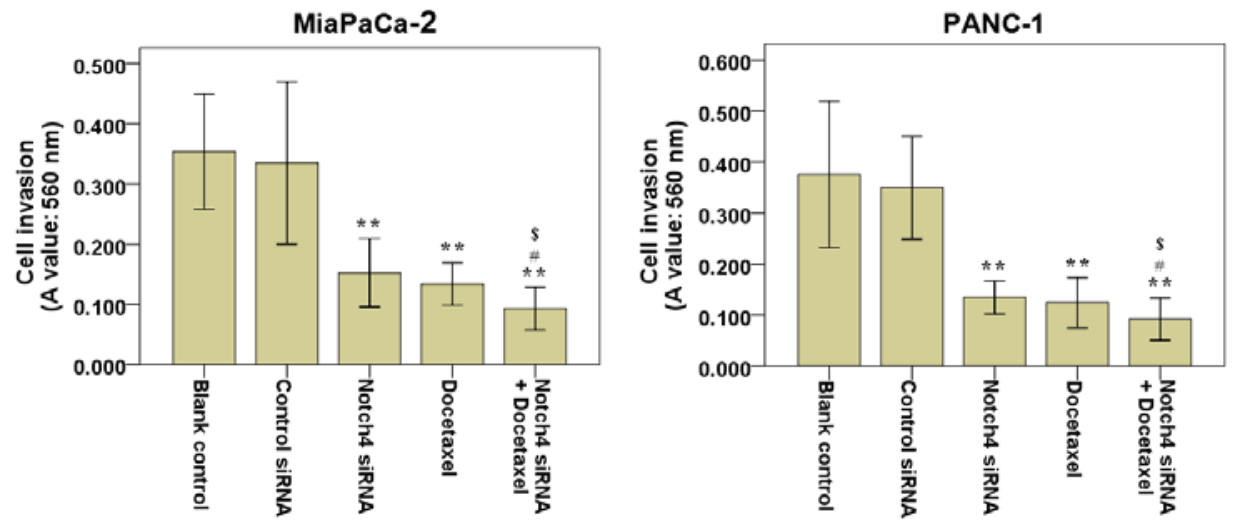

Figure 3. Silencing of Notch4 reduces the cell viability, cell migration and cell invasion abilities of PC cells in vitro. (A) Cells were harvested at days 1-7 post-transfection, and cell viability was measured by 3-(4,5-dimethylthiazol-2-yl)-2,5-diphenyltetrazolium bromide assay. Decreased cell viability was detected in Notch4 siRNA-transfected cells compared with that of the blank control and control siRNA cells. (B) PC cells were treated as described, and cell migration assays were performed. The migrated cells on the lower side of the filter were fixed and stained with 4',6-diamidino-2-phenylindole. The mean number of migrated cells in $\geq 5$ visual fields of three independent experiments was calculated. (C) Representative experiments of cell migration assays are shown (magnification, x200). (D) PC cells were treated as described, and cell invasion assays were performed. The invaded cells were stained and eluted for absorbance readings at $560 \mathrm{~nm}$. Data are the mean \pm standard deviation of $\geq 2$ independent experiments $\left({ }^{*} \mathrm{P}<0.05\right.$, ${ }^{* * *} \mathrm{P}<0.01$ vs. control siRNA; ${ }^{\text {}} \mathrm{P}<0.05$ vs Notch4 siRNA alone; ${ }^{\$} \mathrm{P}<0.05$ vs. docetaxel alone). PC, pancreatic cancer; siRNA, small interfering RNA; A, absorbance. 

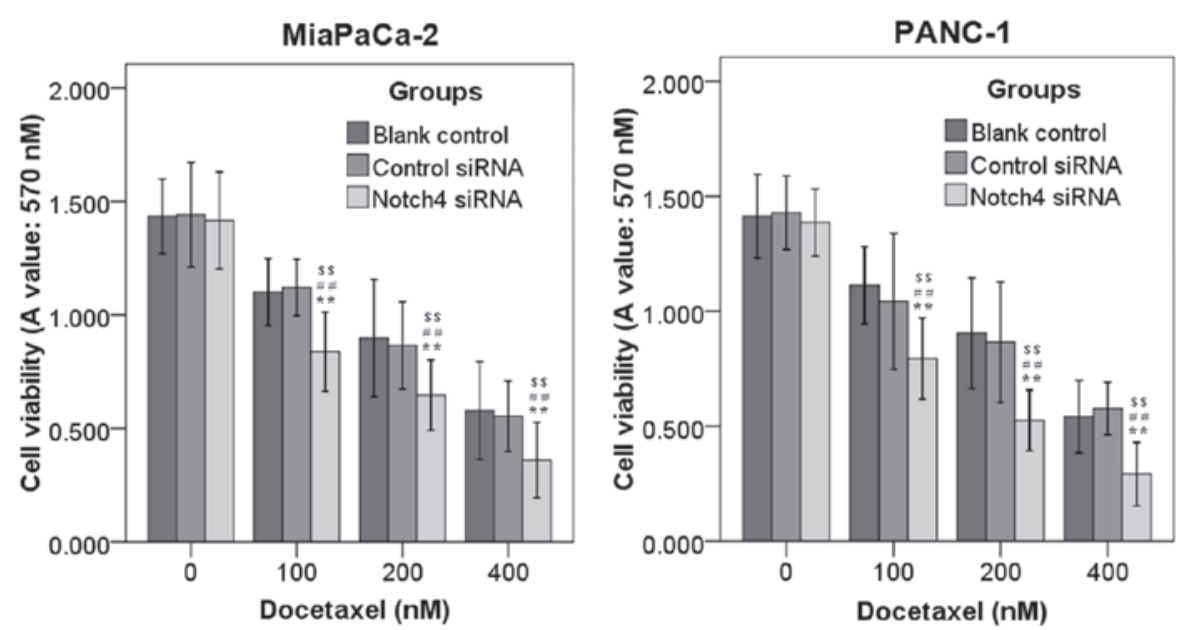

Figure 4. Silencing of Notch4 enhances the sensitivity of pancreatic cancer cells to docetaxel treatment. MiaPaCa-2 and PANC-1 cells transfected with control siRNA or Notch4 siRNA were treated with various concentrations of docetaxel. Cell viability was determined by 3-(4,5-dimethylthiazol-2-yl)-2,5-diphenyltetrazolium bromide assay. Data are the mean \pm standard deviation of $\geq 2$ independent experiments ${ }^{* *} \mathrm{P}<0.01$ vs. control siRNA; ${ }^{\# \#} \mathrm{P}<0.01$ vs. Notch4 siRNA alone; ${ }^{\$ S} \mathrm{P}<0.01$ vs. docetaxel alone). siRNA, small interfering RNA; A, absorbance.
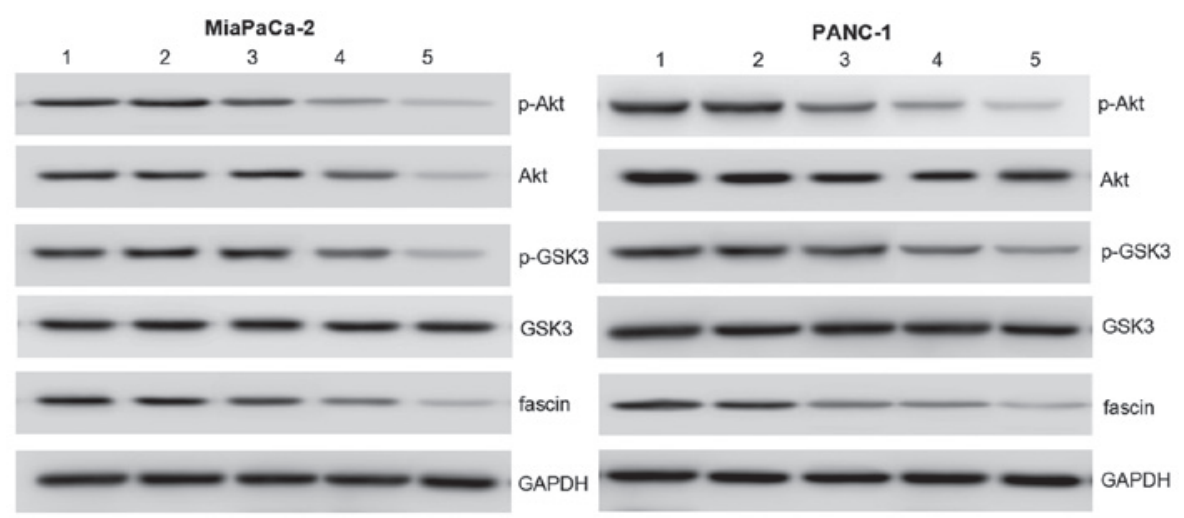

Figure 5. Notch4 siRNA regulates the expression of p-Akt, Akt, GSK3, p-GSK3 and fascin in pancreatic cancer cells. The protein expression levels were determined by western blot analysis. Akt, p-Akt and fascin protein expression were markedly decreased in the Notch4 siRNA + docetaxel group compared with that in the Notch4 siRNA and docetaxel groups. GAPDH was used as an internal control to demonstrate equal protein loading. Lane 1, blank control; lane 2, control siRNA; lane 3, Notch4 siRNA; lane 4, docetaxel; lane 5, Notch4 siRNA + docetaxel. GSK3, glycogen synthase kinase 3; GAPDH, glyceraldehyde 3-phosphate dehydrogenase; p, phosphorylated; siRNA, small interfering RNA.

that Notch4 expression was significantly upregulated in PC cell lines compared with that in an immortalized but not transformed pancreatic epithelial cell line, and RNAi was used to directly downregulate the expression of Notch4 in PC cells. Consistently, further observations revealed that silencing of Notch4 could impede PC cell viability in vitro.

Notch expression in PC was correlated with serosal invasion, lymph node metastasis, histopathological grading, tumor-node-metastasis stage and recurrence (21-24). Enhanced Notch4 expression in salivary adenoid cystic carcinoma may stand as a novel mechanism for promoting metastasis (25). Consistently, the present study demonstrated that Notch4 depletion significantly decreased the cell migration and invasion abilities of PC cells. These findings support a regulatory function for the Notch4 molecule in PC cell tumorigenesis through modulating cell migration and invasion in vitro.

Docetaxel is a monocyclic monoterpene with a lemon-like odor and is a major constituent in several citrus oils (26). Previous studies have demonstrated that docetaxel has a chemoprotective effect on several types of tumors $(27,28)$. However, drug resistance is one of the main causes of docetaxel treatment failure $(29,30)$. In the present study, Notch4 siRNA significantly enhanced the inhibition of cell migration and the invasion ability induced by docetaxel in PC cells. This result indicated that the downregulation of Notch4 enhanced the sensitivity of PC cells to docetaxel treatment, suggesting that Notch4 may be an adjuvant gene therapy target to chemotherapy of PC.

Akt is a downstream target of Notch1 signaling, and a significant reduction in cell viability and an increase in apoptosis in PC cells have been correlated with the downregulation of Notch1 and Akt (31). In the present study, it was observed that the downregulation of Notch4 inhibited the activation of Akt, suggesting that Akt signaling may be involved in the anti-tumor effects induced by Notch4 siRNA. Dysregulation of Akt was involved in docetaxel resistance (32). In the present study, it was demonstrated that the downregulation of Notch4 enhanced the inhibition of Akt induced by docetaxel. 
Therefore, it is possible that Akt activation could contribute to the effects of Notch4 knockdown on cell survival and chemotherapy sensitivity. GSK3 is one of the downstream targets of Akt, and the activation of Akt leads to the phosphorylation of GSK3 $(31,32)$. In the present study, in agreement with the decreased phosphorylation of Akt upon Notch4 knockdown, GSK3 phosphorylation was also decreased by Notch4 knockdown.

Of note, it was observed that the downregulation of Notch4 reduced the expression of fascin. Several studies reported that fascin significantly increases cell migration by enhancing the directional motility of cells (33). The present study demonstrated that the downregulation of Notch4 decreased the expression of fascin, suggesting that Notch4 may regulate cell migration, at least by fascin. In addition, fascin is involved in the chemotherapeutic resistance of breast cancer cells via the Akt pathway (34). However, the mutual association between Akt and fascin in PC is still unclear, and requires in-depth study.

In summary, by using RNAi technology, the expression of the Notch4 gene was successfully downregulated, and Notch4 depletion effectively suppressed PC cell viability, migration and invasion abilities, suggesting that silencing of Notch4 inhibits PC growth and metastasis. It was also demonstrated that Notch4 depletion increased the sensitivity to docetaxel treatment of PC cells, and increased the expression and/or activation of Akt and fascin, which may be related to the sensitivity of PC cells to docetaxel treatment. These findings may at least partially imply that Notch4 may represent an appropriate target for tumor therapy of PC. Further studies are necessary to define the exact role of Notch4 on metastasis and chemotherapy resistance in PC.

\section{Acknowledgements}

The present study was supported by grants from the Natural Science Foundation of Zhejiang Province (Zhejiang, China; grant no. LY16H160033), Public Welfare Technical Applied Research Project of Zhejiang Province (Zhejiang, China; grant no. 2016C33189), Science and Technology Plans of Taizhou City (Taizhou, China; grant no. 1301KY39), Science and Technology Plans of Jiaojiang District of Taizhou City (Taizhou, China; grant no. 132061) and National Innovation and Entrepreneurship Training Project for University Students (Beijing, China; grant no. 201410350020).

\section{References}

1. Teague A, Lim KH and Wang-Gillam A: Advanced pancreatic adenocarcinoma: A review of current treatment strategies and developing therapies. Ther Adv Med Oncol 7: 68-84, 2015.

2. Ryan DP, Hong TS and Bardeesy N: Pancreatic adenocarcinoma. N Engl J Med. 371: 1039-1049, 2014.

3. Yeo TP: Demographics, epidemiology, and inheritance of pancreatic ductal adenocarcinoma. Semin Oncol 42: 8-18, 2015.

4. He XY and Yuan YZ: Advances in pancreatic cancer research: Moving towards early detection. World J Gastroenterol 20: 11241-11248, 2014.

5. Yilmaz M and Christofori G: Mechanisms of motility in metastasizing cells. Mol Cancer Res 8: 629-642, 2010.

6. Qian C, Liu F, Ye B, Zhang X, Liang Y and Yao J: Notch4 promotes pancreatic cancer growth through activation of Wnt1/ $\beta$-catenin signaling. Mol Cell Biochem. 401: 165-174, 2015.

7. Naik S, MacFarlane M and Sarin A: Notch4 signaling confers susceptibility to TRAIL induced apoptosis in breast cancer cells J Cell Biochem 116: 1371-1380, 2015.
8. Ahn S, Hyeon J and Park CK: Notch1 and Notch4 are markers for poor prognosis of hepatocellular carcinoma. Hepatobiliary Pancreat Dis Int 12: 286-294, 2013.

9. Kim SH and Singh SV: The role of polycomb group protein Bmi-1 and Notch4 in breast cancer stem cell inhibition by benzyl isothiocyanate. Breast Cancer Res Treat 149: 681-692, 2015.

10. Manning BD and Cantley LC: AKT/PKB signaling: Navigating downstream. Cell 129: 1261-1274, 2007.

11. Stegeman H, Span PN, Kaanders JH and Bussink J: Improving chemoradiation efficacy by PI3-K/AKT inhibition. Cancer Treat Rev 40: 1182-1191, 2014

12. Hafsi S, Pezzino FM, Candido S, Ligresti G, Spandidos DA, Soua Z, McCubrey JA, Travali S and Libra M: Gene alterations in the PI3K/PTEN/AKT pathway as a mechanism of drug-resistance (review). Int J Oncol 40: 639-644, 2012.

13. Brown KK, Montaser-Kouhsari L, Beck AH and Toker A: MERIT40 is an Akt substrate that promotes resolution of DNA damage induced by chemotherapy. Cell Rep 11: 1358-1366, 2015.

14. Ramakrishnan G, Davaakhuu G, Chung WC, Zhu H, Rana A, Filipovic A, Green AR, Atfi A, Pannuti A, Miele L and Tzivion G: AKT and 14-3-3 regulate Notch4 nuclear localization. Sci Rep 5: 8782, 2015.

15. Zhao X, Gao S, Ren H, Sun W, Zhang H, Sun J, Yang S and Hao J: Hypoxia-inducible factor-1 promotes pancreatic ductal adenocarcinoma invasion and metastasis by activating transcription of the actin-bundling protein fascin. Cancer Res 74: 2455-2464, 2014.

16. Sheppard K, Kinross KM, Solomon B, Pearson RB and Phillips WA: Targeting PI3 kinase/AKT/mTOR signaling in cancer. Crit Rev Oncog 17: 69-95, 2012.

17. Zhou SL, Zhou ZJ, Hu ZQ, Li X, Huang XW, Wang Z, Fan J, Dai Z and Zhou J: CXCR2/CXCL5 axis contributes to epithelial-mesenchymal transition of HCC cells through activating PI3K/Akt/GSK-3 $3 /$ Snail signaling. Cancer Lett 358: 124-135, 2015.

18. Booth AK and Gutierrez-Hartmann A: Signaling pathways regulating pituitary lactotrope homeostasis and tumorigenesis. Adv Exp Med Biol 846: 37-59, 2015.

19. Chen W, Zhang H, Wang J, Cao G, Dong Z, Su H, Zhou X and Zhang S: Lentiviral-mediated gene silencing of Notch-4 inhibits in vitro proliferation and perineural invasion of ACC-M cells. Oncol Rep 29: 1797-1804, 2013.

20. Nagamatsu I, Onishi H, Matsushita S, Kubo M, Kai M, Imaizumi A, Nakano K, Hattori M, Oda Y, Tanaka M and Katano M: NOTCH4 is a potential therapeutic target for triple-negative breast cancer. Anticancer Res 34: 69-80, 2014

21. Singh D, Upadhyay G, Srivastava RK and Shankar S: Recent advances in pancreatic cancer: Biology, treatment, and prevention. Biochim Biophys Acta 1856: 13-27, 2015.

22. Damaskos C, Karatzas T, Kostakis ID, Nikolidakis L, Kostakis A and Kouraklis G: Nuclear receptors in pancreatic tumor cells. Anticancer Res. 34: 6897-6911, 2014.

23. Tang SC and Chen YC: Novel therapeutic targets for pancreatic cancer. World J Gastroenterol 20: 10825-10844, 2014.

24. Mann CD, Bastianpillai C, Neal CP, Masood MM, Jones DJ, Teichert F, Singh R, Karpova E, Berry DP and Manson MM: Notch 3 and HEY-1 as prognostic biomarkers in pancreatic adenocarcinoma. PLoS One 7: e51119, 2012.

25. Ding LC, She L, Zheng DL, Huang QL, Wang JF, Zheng FF and Lu YG: Notch-4 contributes to the metastasis of salivary adenoid cystic carcinoma. Oncol Rep 24: 363-368, 2010.

26. Jakate AS, Einhaus CM, DeAnglis AP, Retzinger GS and Desai PB: Preparation, characterization, and preliminary application of fibrinogen-coated olive oil droplets for the targeted delivery of docetaxel to solid malignancies. Cancer Res 63: 7314-7320, 2003.

27. Iqbal S, Lenz HJ, Gandara DR, Shibata SI, Groshen S, Synold TW and Newman EM: A phase I trial of oxaliplatin in combination with docetaxel in patients with advanced solid tumors. Cancer Chemother Pharmacol 72: 85-91, 2013.

28. Salazar R, Morales S, Gil-Martín M, Aguirre E, Oaknin A, Garcia M, Callies S, Wickremsinhe ER, Benhadji KA and Llombart A: Phase 1 dose escalation and pharmacokinetic evaluation of oral gemcitabine prodrug (LY2334737) in combination with docetaxel in patients with advanced solid tumors. Cancer Chemother Pharmacol 73: 1205-1215, 2014.

29. Zu S, Ma W, Xiao P, Cui Y, Ma T, Zhou C and Zhang H: Evaluation of docetaxel-sensitive and docetaxel-resistant proteomes in PC-3 cells. Urol Int 95: 114-119, 2015.

30. Mahon KL, Lin HM, Castillo L, Lee BY, Lee-Ng M, Chatfield MD, Chiam K, Breit SN, Brown DA, Molloy MP, et al: Cytokine profiling of docetaxel-resistant castration-resistant prostate cancer. Br J Cancer 112: 1340-1348, 2015 
31. Wang Z, Li Y, Ahmad A, Banerjee S, Azmi AS, Kong D, Wojewoda C, Miele L and Sarkar FH: Downregulation of Notch-1 is associated with Akt and FoxM1 in inducing cell growth inhibition and apoptosis in prostate cancer cells. J Cell Biochem 112: 78-88, 2011.

32. Hour TC, Chung SD, Kang WY, Lin YC, Chuang SJ, Huang AM, Wu WJ, Huang SP, Huang CY and Pu YS: EGFR mediates docetaxel resistance in human castration-resistant prostate cancer through the Akt-dependent expression of ABCB1 (MDR1). Arch Toxicol 89: 591-605, 2015.
33. Sonego M, Gajendra S, Parsons M, Ma Y, Hobbs C, Zentar MP, Williams G, Machesky LM, Doherty P and Lalli G: Fascin regulates the migration of subventricular zone-derived neuroblasts in the postnatal brain. J Neurosci 33: 12171-12185, 2013.

34. Ghebeh H, Al-Khaldi S, Olabi S, Al-Dhfyan A, Al-Mohanna F, Barnawi R, Tulbah A, Al-Tweigeri T, Ajarim D and Al-Alwan M: Fascin is involved in the chemotherapeutic resistance of breast cancer cells predominantly via the PI3K/Akt pathway. Br J Cancer 111: 1552-1561, 2014. 